\title{
Research on event perception based on geo-tagged social media data
}

\author{
Ruoxin Zhu ${ }^{\mathrm{a}, *}$, Chenyu Zuo ${ }^{\text {a }}$, Diao Lin $^{\text {a }}$ \\ ${ }^{a}$ Chair of Cartography, Technical University of Munich, 80333 Munich, Germany, ruoxin.zhu@tum.de, chenyu.zuo@tum.de, \\ diao.lin@tum.de \\ * Corresponding author
}

\begin{abstract}
Technological advancement makes information dissemination more convenient. When a notable event occurs, social media serves a popular platform for citizens to share event-related information. Therefore, in the information age, how to effectively observe the event and improve event management ability is an open question worthy of attention. Traditional social survey methods and various automatic sensors have been widely used to monitor the specific event. However, widely used social media service provides a unique approach for the event study with individuals as smart sensors. How to perceive an event through social media data has triggered a series of researches. Currently, we can find when, where what happened and induced impact based on geo-tagged social media data. However, event study based on social media is still in its infancy. This paper provides an overview of event study based on geo-tagged social media data. Firstly, we introduce the event model and the characteristics of social media data. Then, how to detect and trace event, how to analyze event impact and visually express obtained knowledge are discussed respectively. Subsequently, based on the existing researches, we propose further questions and conclude.
\end{abstract}

Keywords: geo-tagged social media data, social sensing, event detection, event tracking, event impact

\section{Motivation}

The world has witnessed a tremendous upsurge of the event which began during the late nineteenth century and has continued right into the twenty-first century (Getz and Page 2016). Various events, especially negative ones (e.g., terrorist attacks, violent incidents), have considerable impacts on a variety of aspects regarding individuals and the society which are worthy of recognition as a distinct academic discipline.

The event-oriented study is all-encompassing and interdisciplinary. The interconnections between various areas of study should foster interdisciplinary theory focused on events to better understand why they exist/occur, and how we can promote positive outcomes as well as minimizing undesirable and unforeseen consequences.

Geo-tagged social media data is a typical social sensing data, which could help to perceive the public's knowledge about their surrounding events directly. Just as the air quality sensor can detect air pollution and the seabed vibration pressure sensor can perform tsunami warning, the reaction of intelligent human beings on social media platform due to the stimuli of the event could help to monitor the event (Goodchild 2007).

So many researches have been conducted to explore the capacity of geotagged social media data for the event perception. In this paper, we review these advances. After the introductory Section 2 detailing event model and the characteristics of geotagged social media data, we enter sections dedicated to advances in event detection (Section 3.1), event tracking (Section 3.2), analysis of event impact
(Section 3.3) and event-oriented visualization (Section 3.4). Section 4 then give an overview of the challenges ahead and future work. Section 5 concludes the paper.

\section{Event meet geo-tagged social media data}

We discuss the characteristics of the event and geo-tagged social media data separately and explore the potential of applying geo-tagged social media to perceive an event.

\subsection{Event-based model}

Different scientists have different interpretations of events because of different perspectives (Casati and Varzi 2008). However, some core features are recognized: a noteworthy occurrence, a unique set of circumstances and a specific place and time. Therefore, to explain any event well enough, we should consider its objective, participants, its place in space and time, its results and the various relationships involved.

Traditional spatiotemporal data models (e.g., snapshot modeling, ground state correction model) lacks the description of the process of change and the cause of change, making it difficult to describe the event. For eventoriented models, the change should be the central concept that modeled. How to build the event-oriented spatiotemporal data model have led to continuous attention. Claramunt and Theriault (1995) and Wang et al. (2005) modeled events as a set of processes changing the state of an entity, thus building the connection between events and spatial object entities. Wu et al. (2008) proposed an improved event-process-based spatiotemporal data model, considering that events are composed of processes and discussing the relationship between events, processes and 
space objects. Liu et al. (2015) proposed an event-based double-sequence spatiotemporal data model, which associates event lists with change lists of spatiotemporal objects. This model not only realizes the mapping of the event to induced state changes of the spatial object but also can describe a series of state change caused by multiple events for spatial object individuals. Polous (2016) depicted an event-based conceptual model and discussed in depth the relationships between events, sub-events, processes and objects, and their related attribute characteristics. Moreover, humans, as important spatiotemporal objects, are included in this space-time model.

Indeed, most current event models consider purely geographic events and mostly ignore human involvement. Exploring the relationship between social events and human behavior is an interesting topic worthy of scrutiny in event modeling. As people's awareness goes deeper, the event-oriented model will be further optimized.

\subsection{Geo-tagged social media data with citizens as social sensors}

The innovation of internet communication technology in the last decades have changed the way citizens communicate. Virtual communities and networks have become a mainstream platform for people to share news and perceptions.

Social media platforms cover a large number of users, with 3.196 billion people worldwide in 2018 enjoying social media service (Chaffey 2019). A large number of users generate a tremendous amount of data. Various social media platforms rich in ample content (e.g., texts, images) and metadata information (e.g., user information, georeference, timestamp). This large amount of data covers various topics, e.g., politics, education, sports, music, fashion, work, food, traveling, entertainment and so on. Also, the social media platform provides a wide range of application programming interfaces for research, corporate and government applications. Those data are free to download within a certain speed and amount. However, the quality of user-generated content varies from excellent to abuse and spam. Therefore, data cleaning is essential for various application purposes.

Several key features of geotagged social media data make it valuable for studying event. Firstly, the real-world event is one of the main driving forces for people to share their knowledge, such as government election and traffic jam. Social media services make it easy for information dissemination. Secondly, the large population and broad geographical distribution of users facilitate the perception of events. Thirdly, the embedded spatial-temporal and semantic information provide valuable indicators to cognize the events. Finally, available data access channels make it possible for event study and further enterprise applications.

\section{Event study meets geo-tagged social media data}

Geo-tagged social media data has been used to study event from multiple aspects. The current focus is mainly on answering when where what happened and induced impact. In this section, we provide an in-depth investigation from the perspectives of event detection, event tracking, analysis of event impact and event-oriented visualization methods.

\subsection{Event detection}

The primary objective of event detection is to identify events using interdisciplinary techniques. According to the entity type characteristics of the research object, event detection methods can be concluded into two categories. One is the detection method based on Twitter-like textual streams, which is more focusing on the analysis of textual feature. Another is a detection method based on the Flickrlike multimedia streams, considering visual, textual, and spatiotemporal features for multimodal fusion.

\subsubsection{Event detection from Twitter-like textual streams}

There are two types of solutions for event detection based on Twitter-like textual streams: document-pivot detection and feature-pivot detection. The document-pivot detection method focuses on the integration of documents, while the feature-pivot detection method focuses on bursty features clustering from the documents stream.

The core of document-pivot detection approach is to cluster documents related to the same event to discover potential events. Moreover, according to the degree of semantic considerations, the typical methods adopted can be summarized into three categories: Vector Space Model (VSM), Latent Semantic Analysis (LSA) and Latent Dirichlet Allocation (LDA).

One limitation about VSM-based method is that it will result in low computational efficiency when dealing with a high-dimensional sparse matrix. The LSA-based method can reduce the dimensionality of term vectors and choose the most informative terms. The basic idea of the LSA algorithm is to map text from sparse high-dimensional lexical space to low-dimensional latent semantic space and compute similarity in latent semantic space. Nevertheless, it is hard to distinguish different events related to the same topic. Therefore, both spatial-temporal and textual information could be jointly combined to improve the performance in document clustering.

The LDA model is a three-layer Bayesian probability topic model with the three-layer structure of words, topics, and documents. This probabilistic model is usually adapted to cope with the characteristics of social media data (e.g., short message) or extended to integrated spatial and temporal features for event detection. For example, Zhang et al. (2017) modeled the text, time, location of tweets as multimodal embedding and utilized various features to explore the possible geo-topics via a Bayesian mixture model. A regression classifier was further applied to identify real local events from candidate geo-topics. Also, some scholars explore various auxiliary methods to improve the efficiency of event clustering. Valkanas and Gunopulos (2013) employed sentiment analysis to detect events from Twitter streams. The targeted event detection will pay attention to the area where a surge in any emotional state is observed. When investigating a specific 
event type, supervised learning classifiers can be used to filter tweets which are not related to the specific event (Sakaki et al. 2010).

Feature-pivot detection methods are more suitable for realtime event detection. Its core objective is to find bursty features from social media streams and cluster them. It consists of three main steps: feature extraction, feature selection, and feature clustering. Various features are available for feature extraction, such as unigrams, bigrams, and n-grams. The bursty features which could be identified as potential event entities are selected from a large number of extracted features based on various selection techniques (e.g., discrepancy principle, wavelet analysis, and Discrete Fourier Transform). Finally, clustering algorithms (e.g., model-based, graph-based, and density-based clustering algorithms) could be used to cluster the bursty features into potential events.

As the tweet number related to local events are relatively small, frequency-based approaches alone are not capable of event detection. Location information of social media data is further integrated to identify and detect local events. Sugitani et al. (2013) applied a hierarchical clustering method to cluster the tweets based on the geo-distance and identify the spatial and temporal burstiness of terms in each cluster to identify real local events. Krumm and Horvitz (2015) split the space into hierarchical and relatively small size regions and utilized the regression errors of geotagged tweet volumes in each volume to search local event regions. Based on the spatial concentration analysis of temporal keywords, local event information could be detected in real-time from Twitter stream.

\subsubsection{Event detection from Flickr-like multimedia streams}

Supervised methods and unsupervised methods are all widely adopted in dealing with event detection from Flickr-like social media streams.

Early machine learning-based detection methods only focused on the image itself. Based on events-related image data set, event classifier will be built, and new images can be categorized into a specific known event type. To further improve the capability of event detection, multi-modal fusion is adopted to improve classification accuracy. Petkos et al. (2012) considered time, space, and visual features, proposing a standard multimodal spectral clustering algorithm. This method train classifier as an indicator matrix to supervise the multimodal fusion and clustering. Ahsan et al. (2017) proposed a web-based supervised learning approach to learn visual concepts relevant to specific event categories.

On the one hand, Wikipedia and Flickr tags were used to mine event concept with Google News Dataset as additional resources. On the other hand, Microsoft Bing was used to searching and build event-related training dataset. Classifiers were trained with deep CNN features extracted from a pre-trained network on all the images. This Internet-based learning method requires only a few labeled examples and can achieve ideal results outperform state of the art.
In addition to progress in supervised learning, research on unsupervised learning-based event detection is continuing. Papadopoulos et al. (2011) built hybrid image-similarity graphs based on image and tag similarity. Considering visual features (speeded up robust features and vector of locally aggregated descriptors), text (title, description, and tags) and time, Schinas et al. (2015) used a sliding timewindow to construct similarity graph and applied a graph clustering algorithm to detect events. Fully consider multiple heterogeneous features (location, user identity time, visual feature and tags), Yang et al. (2017) proposed a three-stage framework for event detection. First, the multimodal fusion model combined soft-voting strategy and graph random walk model was used to obtain fused features. Then, the dual structure constrained multimodal feature coding model was used to conduct multimodal data representations. In the end, hybrid clustering models were applied to discover potential events. The comparative test shows that four clustering methods (DBSCAN, K-Means and its two semi-supervised methods) based on the proposed framework can achieve better performance than state of the art. Moreover, the two semi-supervised clustering methods perform better.

\subsection{Event tracking}

The primary purpose of event tracking is to discover unlabeled stories about the detected or known event. With geo-tagged social media data, we can retrieve eventrelated information, and understand the evolution of events.

\subsubsection{Extraction of event-related information}

To further analyze and recognize interesting event after event detection, information extraction algorithm is needed to obtain more event-related information from the original data. In addition to the traditional keyword retrieval methods, researchers also tried other strategies to make further improvements. Abel et al. (2012) proposed a user-driven semantic filtering strategy for real-world incidents or crises tracking. Murzintcev and Cheng (2017) proposed an automated process for the collection of hashtags related to an interesting event, thereby obtaining event related information. Regarding the extraction of flood-related information, Feng and Sester (2018) studied text-based and photo-based classification respectively and performed performance tests on various classification algorithms (e.g., random forest, ConvNets). The optimal classifiers were used to extract the flood-related text and photos respectively. This method is also applicable to the extraction of other categories of events.

\subsubsection{Event tracking based semantic evolution}

Some scholars try to understand the event by observing semantic evolution. Osborne et al. (2014) designed a realtime event tracking and summarization method with the sliding window method. For a given event, the search query will be formed on the most informative terms (nouns, adjectives, verbs, and cardinal numbers). When new related tweets are coming, redundancy tweets will be filtered and ranked tweets will be used to form an updated summary of the event by performing extractive summarization. Weiler et al. (2014) used the most co- 
occurrence words extracted from Twitter streams to describe events. The co-occurrence terms were ordered continuously, and the occurring event was summarized with top n co-occurrence terms. Cai et al. (2015) used a generative probabilistic model for event detection. Based on this method, maximum-weighted bipartite graph matching was used to trace the event evolution along the temporal dimension. The case study based on event "Snowden" shows that this method can effectively track the progress of event with time series. The graph-based clustering algorithm was applied periodically to detect dense sub-graphs that correspond to events with considering the features of time, text and image (Schinas et al. 2015). For event summarization, representative and diverse sub-set of images will be selected based on a graph-based ranking algorithm.

\subsubsection{Event tracking based spatial-temporal evolution}

Geo-tagged social media data can also describe the event evolution from a spatial-temporal perspective. Zhou and $\mathrm{Xu}$ (2017) used time spectrum to find the start, end, or prime time of the event. Based on the density of tweets, contour lines were built to simulate the spatial pattern of events. Based on spatial densities of both event-related posts and all posts, Gao et al. (2018) adopted Epanechnikov kernel function to design social media event rate (SMER) maps for tracking spatial and temporal trends of the event. This study used SMER maps to estimate local event baseline and reveal potential spatiotemporal patterns. Also, geotagged photos can be used to monitor scene changes. Yan et al. (2017) proposed a workflow to monitor and assess post-disaster recovery based on geo-tagged Flickr photos. A space-time bin method in both spatial and temporal dimensions was used to assess the recovery of scenic spots by comparing the similarities of tourist photos in the affected areas at different periods. Timely tracking disaster recovery is beneficial to disaster management and visitor awareness.

\subsection{Analysis of event impact}

Timely analysis of event impact helps to improve situational awareness and to understand public opinions, which can help the government or enterprise take necessary measures to amply/reduce the positive/negative impact. At present, many scholars have carried out much work to understand the impact of events from geo-tagged social media data.

\subsubsection{Situational awareness}

One of the leading service capabilities of geotagged social media data is situational awareness about the impact of the events. To understand its application ability for emergency management professionals, MacEachren et al. (2011) conducted a series of questionnaires. The results show that mainstream social platforms (e.g., Facebook, LinkedIn, Twitter) are all used commonly in personal use or professional purposes. Moreover, Maps, photos/video collections, time graphs are the top three expected tools and features of the web-based application for emergency management.
Crooks et al. (2013) studied the perception of tweet data on earthquakes. Although it is not possible to monitor the intensity of an earthquake like a seismograph, the tweetlike social perception system is used by the rapid identification and localization of the impact area of the event. Similar researches include flood perception studies, fire perception studies, and storm perception studies. Related photos attached to tweets can provide a clear idea of a disaster situation (Dashti et al. 2014).

In addition to natural disasters, influenza also could be monitored by geo-tagged social media data. Using support vector regression, Signorini et al. (2011) implemented the collection of influenza-related tweets and monitoring of actual disease activity. Gao et al. (2018) analyzed the spread of influenza in real-time based on twitter. Case study during the 2013-2014 flu season in the United States shows that the proposed procedure yields results that correlate strongly with national and local influenza-like illness (ILI) reports.

\subsubsection{Public opinion mining}

Besides situational awareness, we can also use geo-tagged social media data to analyze the impacts of the event on citizens.

Caragea et al. (2014) performed sentiment analysis during Hurricane Sandy and used map-based visual representation to show the relationship between users' sentiments change with the relative distance from the disaster. Zhou and Xu (2017) analyzed the ability of social media data to perceive people's emotions about the specific event. Regarding political opinion, Tumasjan et al. (2010) investigated whether Twitter could be used as a valid indicator mirror offline political sentiment. A case study on the German federal election shows its feasibility. Jahanbakhsh and Moon (2014) studied the 2012 US presidential election combined with machine learning and LDA model. The opinion mining from geo-tagged social media data is consistent with actual public opinion, showing that it is an effective way to understand social attitudes toward event through social media.

\subsection{Event-oriented visualization}

Graphical presentation of quantitative data can significantly improve information perception, retention, and absorption. In event studies, visualization methods are increasingly being used to display and analyze remarkable results.

\subsubsection{Charts}

Chart, such as line chart, bar chart, and stacked graph, is a classic method to demonstrate the spatiotemporal pattern of event evolution. For example, in the study of tracking and measuring Influenza, Signorini et al. (2011) applied Line charts to describe the temporal pattern of morbidity based related tweets numbers along the time. In a case study based on the Australia 2010-2011 flood, Cheong and Cheong (2011) designed networks to present the main component of users and the responses for particular tweets. This method can help to obtain the overview of users' reposting patterns during the spreading of the news. Guille and Favre (2015) used a bar chart to show temporal 
patterns of multiple topics, which could aid the event detection.

\subsubsection{Mapping}

A map is a vivid form of expression which allows users to explore the regional, spatial-temporal pattern intuitively.

Point-based visualization helps to place individual points within the spatial context. Each point represents an object, and its visual variables (e.g., color, size) carries related information. To track the public concern during the H1N1 pandemic, Signorini et al. (2011) displayed the near-realtime influenza-related tweets in a map. Marcus et al. (2011) applied a streaming algorithm to show the current tweets related to the inputted keywords. The map-based expression depicted the real-time spreading of an event on Twitter.

Line-based visualization turns the discrete data points into a continuous fitted curve and can also be used to depict locations along trajectories. Sakaki et al. (2010) predicted and displayed the trajectory of the typhoon against various simulation methods based on 2037 related tweets. Endarnoto et al. (2011) promoted an information extraction process to obtain traffic information, using different colors to represent the traffic condition of the inquired road segment.

Region-based visualization can be used to depict aggregated information over regions of a predefined granularity. When an overall view of a region needed to be generalized, a general map can be applied. Gao et al. (2018) used heatmap to visualize extracted events from social media data. Huang et al. (2017) aggregated data based on administrative regions, which is convenient for decisionmaking.

\subsubsection{Text visualization}

Visualization of textual data is also an essential way of knowledge dissemination. It can help people better understand event conditions and human opinions. Fisher et al. (2008) developed a system "Narratives" to visualize the extracted event topics from social media data. This system can address those scenarios in a temporal view. Similarly, Zhou and Chen (2014) used streamgraph to present the temporal social events' patterns. Schreck and Keim (2013) applied a node-link-based visualization to give an overview of the news. Word cloud is also widely used in textual data visualization. Zhou and Xu (2017) used the word cloud to depict the keywords in an event. The size of the words stands for the frequency of mentioned topics in social media; the curves between words show the relationships between topic categories. Dörk and Knight (2015) designed an interactive word cloud system, which can allow users to explore the subtopics by their input.

\subsubsection{Integrated system}

To analyze the geospatial, temporal, textual, and other contextual information of the extracted social events, interactive visualization systems are prototyped. Kuang et al. (2014) designed an interactive visualization platform to depict the event. The interface provides the context of an event, such as representative images, word cloud, geographic and temporal summarization, and original micro-posts. Thom et al. (2015) implemented a visualization platform to analyze the 2013 German Flood. In addition to interactive maps and essential query functions, this platform also expresses sentiment volumes and unusual topics. Robinson et al. (2017) developed a system "STempo", supporting visual analysis and pattern discovery in terms of time, location, and content.

\section{Potential and challenges}

Current research works on events in geo-tagged social media are mainly focused on the knowledge discovery about when, where, what happened and induced impact. We acknowledge that there are still many potential areas in this field that can be improved. Based on existing researches, we foresee that event studies based on geotagged social media data will develop in the following several areas:

\subsection{Comprehensive analysis based on multi-source data}

Current research on event study is mainly based on a single data source to carry out method innovation. To improve the accuracy and credibility of event analysis, other types of perception should not be ignored. For example, Li and $\mathrm{Li}$ (2014) used night-light remote sensing data to assess the socio-economic impact of the Syrian civil war. Zhang et al. (2015) designed a routine social activeness model based on human flow dynamics, which can find the time and place of events and measure its scale. This kind of data can play an important role in event study. Event analysis based on multi-source data deserves further attention. Besides, credibility is an important question worth paying attention to. It has been observed that in 2010 U.S. midterm elections, social bots were used to guide the political inclination of the public (Ratkiewicz et al. 2011). Abuse of social bots may cause disastrous consequence. Therefore, how to use multi-source data to acquire reliable result is worth pondering.

\subsection{From phenomena description to knowledge mining and derivative prediction}

The current ability of event perception based on geotagged social media data has already met the basic needs of situational awareness. However, the current analysis still stays in the description of the phenomenon. Knowledge hidden under the event-related information needs to be further discovered. Every notable event could involve some relationships with other events. At present, not limited to related relationships, research on causality continues to grow. On the one hand, the innovation of algorithms (e.g., structural equation model and directed acyclic graph) has been continuing (Burgess and Thompson 2015). On the other hand, application researches in various fields (e.g., biomedicine, climate science) are also underway. Driven by events, in social media people post not only their perceptual knowledge but also their rational knowledge. Based on existing research, we could move forward in relationship mining among objects with events, events with events, and the involving processes, thereby improving the understanding of event ecology. Besides, situational awareness-based event 
prediction needs further exploration. Study on professional prediction model based on situational awareness from social sensing and other various automatic sensors could serve for emergency response and rescue.

\subsection{From visualization to visual storytelling}

As has been widely used in movies and novels, tinging a series of information into stories can effectively present information and memorably make a point. Perhaps visual stories could become an effective way to depict information about the complex event and pass on knowledge in the future. As a carrier of space-time information, maps still have considerable potential for storytelling. Combined with other necessary visual elements, the map can serve as a carrier for the story and the map-led storytelling is fully capable of assisting the audience in following the plot (Caquard and Cartwright 2014). Studying how to use maps to narrate stories could be a potential research hotspot in cartography.

Also, the story is like a series of states and processes that are strung together in time. It usually contains the beginning, middle, and end, and contains the cause and effect. Building a visual story is like literary creation, strive to be vivid and impressive. Ordering and interacting are the core of story construction. How to match different order and interacting structures to achieve better narrative effects, needs continuous attention.

\section{Conclusion}

Thanks to the rapid innovation of technology, the full application of social media service provide a unique social sensing channel to study events. A large user base, extensive geographical distribution, and real-time information dissemination have significantly improved our ability to perceive events.

In this paper, we review the current state of the art in event study with geo-tagged social media data. Firstly, this paper introduces the significance of event study, then describes the event model and the characteristics of geotagged social media data. After that, the current research progress is introduced from several aspects: event detection, event tracking, analysis of event impact and event visualization. Finally, the potential research areas are discussed based on the current researches.

The event study is essential for social sustainability. With the advancement of technology and the deepening of research, essential achievements will play an essential role in government decision-making, business planning, education, to name only a few. Mastery of social sensing helps to intelligently perceive the society in which we live and improve the living environment.

\section{References}

Abel, F., Hauff, C., Houben, G. J., Stronkman, R. and Tao, K. (2012, June). Semantics+ filtering+ search= incident. exploring information in social web streams. In Proceedings of the 23rd ACM conference on Hypertext and social media (pp. 285-294). ACM.
Ahsan, U., Sun, C., Hays, J. and Essa, I. (2017). Complex event recognition from images with few training examples. arXiv preprint arXiv:1701.04769.

Burgess, S. and Thompson, S. G. (2015). Multivariable Mendelian randomization: the use of pleiotropic genetic variants to estimate causal effects. American journal of epidemiology, 181(4), 251-260.

Cai, H., Yang, Y., Li, X. and Huang, Z. (2015, October). What are popular: exploring twitter features for event detection, tracking and visualization. In Proceedings of the 23rd ACM international conference on Multimedia (pp. 89-98). ACM.

Caquard, S. and Cartwright, W. (2014). Narrative cartography: From mapping stories to the narrative of maps and mapping.

Caragea, C., Squicciarini, A. C., Stehle, S., Neppalli, K. and Tapia, A. H. (2014, May). Mapping moods: Geomapped sentiment analysis during hurricane sandy. In ISCRAM.

Casati, R. and Varzi, A. C. (2008). Event concepts. Understanding events: From perception to action, 31-53.

Chaffey, D. (2019). Global social media research summary $2019 . \quad$ Available online: https://www.smartinsights.com/social-mediamarketing/social-media-strategy/new-global-socialmedia-research/ (accessed on 20 February 2019)

Cheong, F. and Cheong, C. (2011). Social Media Data Mining: A Social Network Analysis Of Tweets During The 2010-2011 Australian Floods. PACIS, 11, 46-46.

Claramunt, C. and Thériault, M. (1995). Managing time in GIS an event-oriented approach. In Recent advances in temporal databases (pp. 23-42). Springer, London.

Crooks, A., Croitoru, A., Stefanidis, A. and Radzikowski, J. (2013). \# Earthquake: Twitter as a distributed sensor system. Transactions in GIS, 17(1), 124-147.

Dashti, S., Palen, L., Heris, M. P., Anderson, K. M., Anderson, T. J. and Anderson, S. (2014, May). Supporting disaster reconnaissance with social media data: A design-oriented case study of the 2013 Colorado floods. In ISCRAM.

Dörk, M. and Knight, D. (2015). WordWanderer: a navigational approach to text visualisation. Corpora, 10(1), 83-94.

Endarnoto, S. K., Pradipta, S., Nugroho, A. S. and Purnama, J. (2011, July). Traffic condition information extraction \& visualization from social media twitter for android mobile application. In Electrical Engineering and Informatics (ICEEI), 2011 International Conference on (pp. 1-4). IEEE.

Feng, Y. and Sester, M. (2018). Extraction of pluvial flood relevant volunteered geographic information (VGI) by deep learning from user generated texts and photos. ISPRS International Journal of GeoInformation, 7(2), 39.

Fisher, D., Hoff, A., Robertson, G. and Hurst, M. (2008, October). Narratives: A visualization to track narrative 
events as they develop. In Visual Analytics Science and Technology, 2008. VAST'08. IEEE Symposium on (pp. 115-122). IEEE.

Gao, Y., Wang, S., Padmanabhan, A., Yin, J. and Cao, G. (2018). Mapping spatiotemporal patterns of events using social media: a case study of influenza trends. International Journal of Geographical Information Science, 32(3), 425-449.

Getz, D. and Page, S. (2016). Event studies: Theory, research and policy for planned events. Routledge.

Goodchild, M. F. (2007). Citizens as sensors: the world of volunteered geography. GeoJournal, 69(4), 211-221.

Guille, A. and Favre, C. (2015). Event detection, tracking, and visualization in twitter: a mention-anomaly-based approach. Social Network Analysis and Mining, 5(1), 18.

Huang, W., Fan, H. and Zipf, A. (2017). Towards Detecting the Crowd Involved in Social Events. ISPRS International Journal of Geo-Information, 6(10), 305.

Yan, Y., Eckle, M., Kuo, C. L., Herfort, B., Fan, H. and Zipf, A. (2017). Monitoring and assessing post-disaster tourism recovery using geotagged social media data. ISPRS International Journal of GeoInformation, 6(5), 144.

Yang, Z., Li, Q., Liu, W., Ma, Y. and Cheng, M. (2017). Dual graph regularized NMF model for social event detection from Flickr data. World Wide Web, 20(5), 9951015.

Jahanbakhsh, K. and Moon, Y. (2014). The predictive power of social media: on the predictability of US presidential elections using Twitter. arXiv preprint arXiv:1407.0622.

Krumm, J. and Horvitz, E. (2015, November). Eyewitness: Identifying local events via space-time signals in twitter feeds. In Proceedings of the 23rd SIGSPATIAL International Conference on Advances in Geographic Information Systems(p. 20). ACM.

Kuang, C., Tang, J., Liu, Z. and Sun, M. (2014, May). ImgWordle: image and text visualization for events in microblogging services. In Proceedings of the 2014 International Working Conference on Advanced Visual Interfaces (pp. 371-372). ACM.

Li, X. and Li, D. (2014). Can night-time light images play a role in evaluating the Syrian Crisis?. International Journal of Remote Sensing, 35(18), 6648-6661.

Liu, F., He, Z. W. and Zheng, X. Q. (2015). An eventbased dual-lists spatio-temporal data model. Geography and Geo-Information Science, 3, 001.

MacEachren, A. M., Jaiswal, A., Robinson, A. C., Pezanowski, S., Savelyev, A., Mitra, P., ... and Blanford, J. (2011, October). Senseplace2: Geotwitter analytics support for situational awareness. In Visual analytics science and technology (VAST), 2011 IEEE conference on (pp. 181-190). IEEE.

Marcus, A., Bernstein, M. S., Badar, O., Karger, D. R., Madden, S. and Miller, R. C. (2011, May). Twitinfo: aggregating and visualizing microblogs for event exploration. In Proceedings of the SIGCHI conference on
Human factors in computing systems (pp. 227-236). ACM.

Murzintcev, N. and Cheng, C. (2017). Disaster Hashtags in Social Media. ISPRS International Journal of GeoInformation, 6(7), 204.

Osborne, M., Moran, S., McCreadie, R., Von Lunen, A., Sykora, M. D., Cano, E., ... \& Jackson, T. (2014). Realtime detection, tracking, and monitoring of automatically discovered events in social media.

Papadopoulos, S., Zigkolis, C., Kompatsiaris, Y. and Vakali, A. (2010). Cluster-based landmark and event detection for tagged photo collections. IEEE MultiMedia, (1), 52-63.

Petkos, G., Papadopoulos, S. and Kompatsiaris, Y. (2012, June). Social event detection using multimodal clustering and integrating supervisory signals. In Proceedings of the 2nd ACM International Conference on Multimedia Retrieval (p. 23). ACM.

Polous, K. (2016). Event Cartography: A New Perspective in Mapping (Doctoral dissertation, Technische Universität München).

Ratkiewicz, J., Conover, M., Meiss, M. R., Gonçalves, B., Flammini, A. and Menczer, F. (2011). Detecting and tracking political abuse in social media. ICWSM, 11, 297-304.

Robinson, A. C., Peuquet, D. J., Pezanowski, S., Hardisty, F. A. and Swedberg, B. (2017). Design and evaluation of a geovisual analytics system for uncovering patterns in spatio-temporal event data. Cartography and Geographic Information Science, 44(3), 216-228.

Sakaki, T., Okazaki, M. and Matsuo, Y. (2010, April). Earthquake shakes Twitter users: real-time event detection by social sensors. In Proceedings of the 19th international conference on World wide web (pp. 851860). ACM.

Schinas, M., Papadopoulos, S., Petkos, G., Kompatsiaris, Y. and Mitkas, P. A. (2015, October). Multimodal graphbased event detection and summarization in social media streams. In Proceedings of the 23rd ACM international conference on Multimedia (pp. 189-192). ACM.

Schreck, T. and Keim, D. (2013). Visual analysis of social media data. Computer, 46(5), 68-75.

Signorini, A., Segre, A. M. and Polgreen, P. M. (2011). The use of Twitter to track levels of disease activity and public concern in the US during the influenza A H1N1 pandemic. PloS one, 6(5), e1946.

Sugitani, T., Shirakawa, M., Hara, T. and Nishio, S. (2013, March). Detecting local events by analyzing spatiotemporal locality of tweets. In Advanced Information Networking and Applications Workshops (WAINA), 2013 27th International Conference on (pp. 191-196). IEEE.

Thom, D., Krüger, R., Ertl, T., Bechstedt, U., Platz, A., Zisgen, J. and Volland, B. (2015, April). Can twitter really save your life? A case study of visual social media analytics for situation awareness. In Visualization 
Symposium (PacificVis), 2015 IEEE Pacific (pp. 183190). IEEE.

Tumasjan, A., Sprenger, T. O., Sandner, P. G. and Welpe, I. M. (2010). Predicting elections with twitter: What 140 characters reveal about political sentiment. Icwsm, 10(1), 178-185.

Valkanas, G. and Gunopulos, D. (2013, October). How the live web feels about events. In Proceedings of the 22nd ACM international conference on Information \& Knowledge Management (pp. 639-648). ACM.

Wang, C., Zhang, Jun., Jiang, T. (2005). Implementation research of event-based spatio-temporal data model[J]. Science of Surveying and Mapping, 30(2): 67-69.

Weiler, A., Grossniklaus, M. and Scholl, M. H. (2014). Event identification and tracking in social media streaming data. In EDBT/ICDT (pp. 282-287).

Wu, C. and Lyu, G. (2008). Improved eventprocess based on spatio-temporal model. Geomatics and Information Science of Wuhan University, 33(12), 1250-1254.

Zhang, C., Liu, L., Lei, D., Yuan, Q., Zhuang, H., Hanratty, T. and Han, J. (2017, August). Triovecevent: Embedding-based online local event detection in geotagged tweet streams. In Proceedings of the 23rd ACM SIGKDD International Conference on Knowledge Discovery and Data Mining (pp. 595-604). ACM.

Zhang, W., Qi, G., Pan, G., Lu, H., Li, S. and Wu, Z. (2015). City-scale social event detection and evaluation with taxi traces. ACM Transactions on Intelligent Systems and Technology (TIST), 6(3), 40.

Zhou, X. and Chen, L. (2014). Event detection over twitter social media streams. The VLDB Journal-The International Journal on Very Large Data Bases, 23(3), 381-400.

Zhou, X. and Xu, C. (2017). Tracing the spatial-temporal evolution of events based on social media data. ISPRS International Journal of Geo-Information, 6(3), 88. 